\title{
“PERCEPCIÓN DE LOS ESTUDIANTES DE ENFERMERÍA SOBRE LOS ESTILOS DE LIDERAZGO DE LOS DOCENTES DE LA FACULTAD DE ENFERMERÍA DE LA UNIVERSIDAD NACIONAL SAN LUIS GONZAGA DE ICA"
}

\author{
Rosa Hernández Onofra ${ }^{1, a, c}$, Amanda García Aquije ${ }^{2, a, c}$, Hugo Arroyo Anglas ${ }^{3, b}$ \\ ${ }^{1}$ Facultad de Enfermería de la Universidad Nacional San Luis Gonzaga de Ica, ${ }^{2}$ Hospital Augusto Hernández Mendoza. Ica, Perú. ${ }^{3}$ Hospital Santa María del \\ Socorro. Ica, Perú. \\ ${ }^{a}$ Licenciada en Enfermeria, ${ }^{b}$ Médico Cirujano, ${ }^{c}$ Magister en Salud Pública,
}

\section{RESUMEN}

Objetivo: Determinar la percepción que tienen los estudiantes de enfermería sobre los estilos de liderazgo de los docentes de la facultad de enfermería de la Universidad Nacional "San Luis Gonzaga" de Ica. Material y Métodos: Investigación de diseño descriptivo, de tipo cuantitativo, con enfoque epistemológico empirista inductivo, la muestra estuvo conformada por 200 alumnos y el análisis de los resultados se hizo por contraste con los postulados teóricos usando como instrumento el cuestionario de BASS con 45 resultados poco satisfactorios que se relaciona con el comportamiento de los docentes en su desempeño laboral. Resultados: $81,54 \%$ de los alumnos consideran que sus profesores se muestran amables y confiables, un $12 \%$ que manifestaron estar en total desacuerdo. $65 \%$ manifiestan que sus profesores propicien estímulos y motiven a sus estudiantes a realizar sus tareas. Sobre el buen manejo del proceso de comunicación existente entre los alumnos y sus profesores, $56 \%$ respondió que son escuchados por sus maestros, un $17 \%$ está en desacuerdo y $7 \%$ totalmente en desacuerdo. El $46 \%$ asume que sus profesores se muestran tolerantes ante los errores de sus alumnos, un $11 \%$ está en desacuerdo y otro $11 \%$ en total desacuerdo. El $52,5 \%$ ha manifestado que sus profesores suelen ser optimistas y motivadores en el desempeño laboral y una cifra muy baja $5 \%$ se muestra en desacuerdo. Un $42 \%$ afirma que sus docentes consideran en todo momento las necesidades de sus alumnos, un $11 \%$ manifiesta estar en desacuerdo y un $6 \%$ en total desacuerdo. En cuanto a si los docentes transmiten entusiasmo y confianza, un 52,5\% afirmaron estar en total acuerdo, quedando un 5,5\% en desacuerdo. El 50\% manifiesta estar en total acuerdo que sus docentes desarrollen eficazmente un liderazgo en su desempeño laboral y un 22,5\% manifiestan estar en total desacuerdo. Conclusiones: Existe un buen desempeño laboral del docente de la Facultad de Enfermería, los estudiantes perciben que el liderazgo de los docentes de la Facultad de Enfermería es el Transformacional también se evidencia que existe nudos críticos que deben ser atendidos para dar respuesta satisfactoria a los estudiantes.

Palabras claves: Liderazgo, docente, enfermería.

\section{SUMMARY}

Objective: Determine the perceptions of nursing students on the leadership styles of teachers of nursing faculty of "San Luis Gonzaga" of Ica National University. Material and Methods: Research descriptive design, quantitative, with epistemological approach inductive empiricist, the sample consisted of 200 students and analysis of the results was made by contrast with the theoretical postulates using as an instrument the questionnaire BASS 45 results unsatisfactory that relates to the behavior of teachers in their job performance. Results: $81,54 \%$ of students feel that their teachers is friendly and reliable, $12 \%$ who said they strongly disagree. $65 \%$ report that their teachers encourage stimuli and motivate students to do their jobs. The proper management of the process of communication between students and teachers, $56 \%$ said it are heard by their teachers, $17 \%$ disagree and $7 \%$ strongly disagree. $46 \%$ assume that their teachers are tolerant to errors of his students, $11 \%$ disagree and $11 \%$ strongly disagree. $52,5 \%$ said that their teachers are often 
optimistic and motivators on work performance and very low down $5 \%$ disagrees. $42 \%$ say that their teachers at all times consider the needs of their students, $11 \%$ manifested disagree and $6 \%$ strongly disagree. As to whether teachers convey enthusiasm and confidence, $52,5 \%$ said they were in total agreement, leaving a $5,5 \%$ disagree. $50 \%$ claim to be in total agreement that their teachers effectively develop leadership in their job performance and $22,5 \%$ say they strongly disagree. Conclusions: There is a good working performance professor at the School of Nursing, students perceive that the leadership of the teachers of the School of Nursing is the Transformational also evident that there critical points that must be addressed to give satisfactory answers to students.

Keywords: Leadership, teaching, nursing.

\section{INTRODUCCIÓN}

El tema del liderazgo ha sido considerado tradicionalmente como una de las claves más importantes para el desarrollo eficaz de las organizaciones. Por ello, las instituciones educativas están reconociendo la necesidad de mejorar el desempeño laboral de sus docentes, desarrollando en su quehacer cotidiano la competencia de un liderazgo efectivo que vaya en beneficio de la colectividad, ya que las universidades están convencidas que la variable o el elemento principal para el futuro exitoso de las instituciones educativas recaerá en el liderazgo que se desarrolle en ella.

Esta reflexión ha llevado a muchas organizaciones a reestructurar sus procesos y a emprender grandes esfuerzos en la capacitación del personal a su cargo, desarrollando habilidades y destrezas productivas en el ejercicio profesional. Cabe destacar que la tarea educativa siempre ha llevado implícita la función de liderazgo, puesto que la enseñanza es una actividad humana, donde las personas ejercen su influencia de poder en otras, para desarrollar todas sus potencialidades (1).

Actualmente, existen dos grandes compromisos que deben ser asumir por los docentes, para cumplir con su rol social de educar. Por un lado, responder a las demandas y requerimientos educativos que la sociedad, en constante cambio, le impone; y por otro, suscitar las transformaciones que posibiliten escenarios de autorrealización y de reales oportunidades de vida para las personas a su cargo (2).
En este contexto, la función docente se presenta como una tarea variada y compleja que desafía el profesionalismo de los profesores, motivándolos a ejercer un liderazgo que les permita guiar, motivar y movilizar a los demás participantes del proceso de enseñanza-aprendizaje, para promover efectivos y significativos aprendizajes en sus alumnos para que alcancen su pleno desarrollo personal, en sana y justa convivencia con los demás.

De modo que el docente, dentro del campo de la educación, está llamado asumir la responsabilidad de crear oportunidades para promover el acto educativo, de allí que es tan importante el desarrollar un liderazgo eficaz. Por tal razón, un docente en su rol de líder es fundamentalmente un agente de cambio, que amerita toda la astucia necesaria para que su liderazgo sea efectivo, y promueva cambios significativos y perdurables en sus seguidores (3).

En tal sentido, se espera que los lideres educativos del futuro, desarrollen la competencia de un liderazgo efectivo, en busca de soluciones novedosas e innovadoras, preocupándose porque el trabajo siempre tenga un significado y un propósito para sus seguidores, logrando que el producto final tenga un significado para todos los involucrados en el proceso (3).

El liderazgo es un proceso de permanente readecuación e inherente a toda la vida, ya que busca transformar, a todo momento, las potencialidades de sus colaboradores, desarrollando sus capacidades, motivaciones 
y valores, para mejorar su desempeño académico y por ende su desempeño laboral en el futuro (4).

En síntesis, todo hace pensar que el docente universitario debe ir en busca de un liderazgo que atienda a las exigencias actuales, las cuales demandan más atención al ser humano, más integración, más participación de todos los actores de una organización, donde la pirámide de mando se rompe para dar paso a un trabajo integrado holístico, que considera a todos los miembros de una institución indispensables e importantes para ejercer un buen trabajo grupal (4).

Desde esta perspectiva entonces, quien ejerce la función docente debe proyectar y practicar básicamente en su desempeño laboral los elementos que conforman el liderazgo transformacional. El modelo de liderazgo transformacional propuesto por Bass (5) hace hincapié en actividades tales como creatividad, estimulación intelectual, capacidad para estimular e inspirar a sus seguidores más allá de las expectativas, dándole sentido a cada uno de los procesos y eventos. Es por ello que el líder demuestra su atención y consideración individual al tomar en cuenta a la persona, preocupándose por sus necesidades, apoyando su crecimiento y desarrollo en medio de un clima de armonía y empatía. A continuación se mencionan los factores fundamentales que describen la teoría del Liderazgo Transformacional. Según Bass (5) el líder transformacional se distingue por cinco características básicas, relacionándolas con la labor desempeñada por el docente:

- La consideración individual.

- La estimulación intelectual.

- La motivación inspiracional.

- La tolerancia psicológica.

- La influencia idealizada.

Padilla D. en su trabajo de investigación: "Percepción, de los Docentes, Administrativos y Alumnos sobre el Liderazgo y Cultura
Organizacional en la Universidad Particular "San Juan Bautista", Concluye, entre otros: "En las habilidades de liderazgo de dirección estratégicas, cohesión, negociación y toma de decisiones la respuesta más común es la de Indeterminación. Es decir, los públicos estudiados no reconocen estas habilidades en ellos mismos" (6).

Rodríguez N. en su trabajo de investigación titulado "El Clima Escolar", en Lima, concluye: "La evaluación sistemática del clima debe identificar las características negativas, deficiencias y fuentes de problemas (7).

Por todo lo enunciado anteriormente se hizo conveniente realizar un estudio que responde a las siguientes preguntas de investigación ¿Cuál es la percepción de los estudiantes sobre los estilos de liderazgo de los docentes de la facultad de Enfermería de la Universidad Nacional San Luis Gonzaga de Ica?

En base a los antecedentes y bases teóricas, el presente trabajo de investigación tuvo como objetivo, analizar la percepción que tienen los estudiantes en relación al liderazgo desarrollado por los docentes dentro de la Facultad de Enfermería de la Universidad San Luis Gonzaga de Ica en su desempeño laboral fuera y dentro del aula de clase, lo cual constituyó un valioso aporte teórico referencial ya que permitió develar, a través de la información recogida, la percepción que tienen los estudiantes en relación al liderazgo desarrollado por los docentes, determinando características y aspectos principales del liderazgo que poseen los docentes en los alumnos diferentes ciclos, lo cual permite plantear estrategias encaminadas a mejorar la calidad de la enseñanza.

\section{MATERIAL Y MÉTODOS}

La investigación propuesta corresponde a un Diseño Descriptivo, el cual consiste en la caracterización de un fenómeno con el fin de 
establecer su comportamiento (8). El mismo corresponde a un estudio del Tipo Cuantitativo, basado en un enfoque epistemológico empirista inductivo. La población objeto de estudio estuvo conformada por los estudiantes de la Facultad de Enfermería. La unidad muestral estuvo conformada por estudiantes de todos los ciclos de estudio de la Facultad de Enfermería. Se utilizó para seleccionar su tamaño un muestreo probabilístico, aleatorio simple, quedando conformada por 200 estudiantes de ambos sexos, diferentes edades y diferentes secciones, estudiantes que están matriculados en el año 2012. El cuestionario utilizado para conocer las percepciones que tienen los alumnos de sus profesores respecto al liderazgo ejercido en el desempeño de la función docente, es un cuestionario diseñado por los autores que consta de 40 reactivos tipo Lickert, basado en los factores que conforman el modelo propuesto por Bass (5): carisma, estimulación intelectual, consideración individual, motivación inspiracional, influencia idealizada, tolerancia psicológica, dirigido a medir los patrones de frecuencias para cada ítem:
A - Totalmente de acuerdo
$B$ - De acuerdo
C - Ni de acuerdo ni en desacuerdo
D - En desacuerdo
E - Totalmente en desacuerdo

Para el recojo de información utilizamos un cuestionario que consta de dos partes: La primera es de datos generales para saber el perfil de los encuestados, y la segunda parte con 40 preguntas que es un cuestionario adaptado de los autores, sobre características de los docentes en su desempeño laboral, propuestos en la teoría de BASS, relacionados con el liderazgo transformacional (5). Los resultados obtenidos a nivel general, serán sometidos a análisis estadístico descriptivo, utilizando la herramienta informática denominada SPSS versión de prueba. La validez del instrumento se hizo a nivel local mediante el índice de acuerdo y desacuerdos de 6 expertos, la confiabilidad se encontró en la prueba alfa de Cronbach. Según lo estipulado en la declaración de Helsinki (1975-1983) se preservo la privacidad de los informantes, guardando la confidencialidad y el anonimato para garantizar una información fidedigna, confiable y veraz.

\section{RESULTADOS}

Se analizan los datos recogidos sobre la percepción de los estudiantes encuestados sobre el tipo de liderazgo que poseen los docentes: CARISMA: Según los datos analizados, el $81,54 \%$ de los alumnos encuestados consideran que sus profesores se muestran amables y confiables, cualidad que permite mantener comunicaciones efectivas con sus alumnos y con las personas que le rodean, un $12 \%$ que manifestaron estar en total desacuerdo con esta característica de su docente.

ESTÍMULO INDIVIDUAL: Se evidencia que el $65 \%$ de la población encuestada manifiesta que sus profesores propicien estímulos y motiven a sus estudiantes a realizar sus tareas, creando ideas nuevas, contribuyendo al estímulo intelectual que debe fomentar todo educador en su desempeño laboral.

CONSIDERACIÓN INDIVIDUAL: Sobre el buen manejo del proceso de comunicación existente entre los alumnos y sus profesores, el $56 \%$ respondió que son escuchados por sus maestros cuando lo han necesitado, es decir que son capaces de escuchar, sin embargo que un $17 \%$ está en desacuerdo y $7 \%$ totalmente en desacuerdo porque no percibía esta característica en su docente. Este análisis se infiere en que se debe trabajarnos para que exista un proceso de comunicación en la Facultad de Enfermería. TOLERANCIA PSICOLÓGICA: El $46 \%$ de la población encuestada asume que sus profesores se muestran tolerantes ante los errores de sus alumnos, siendo estas unas de las características que debe poseer todo 
docente en su desempeño laboral. Llama la atención de las respuestas dispersas porque un $11 \%$ está en desacuerdo y otro $11 \%$ en total desacuerdo ya que sus profesores no muestran tolerancia entre ellos, cifra que se recomienda resaltar para tomar medidas al respecto porque esta característica es fundamental para generar una interrelación de confianza que permitirá un mejor desenvolvimiento del individuo. MOTIVACIÓN INSPIRADA: La mayor parte de los estudiantes consideran que sus docentes desarrollan eficazmente un liderazgo en su desempeño laboral, ya que el $52,5 \%$ de los estudiantes lo han manifestado al considerar que sus profesores suelen ser optimistas y motivadores en el desempeño laboral y en una cifra muy baja $5 \%$ se muestra en desacuerdo.

CONSIDERACIÓN

INIDIVIDUAL: Un $42 \%$ afirma que sus docentes consideran en todo momento las necesidades de sus alumnos sin embargo, es necesario destacar que esta cifra debería ser más alta, ya que el rol del líder docente es conocer las características individuales de sus alumnos para poder satisfacer sus necesidades y un $11 \%$ manifiesta estar en desacuerdo y un $6 \%$ en total desacuerdo con esta característica. INFLUENCIA IDEALIZADA: En cuanto a si los docentes transmiten entusiasmo y confianza, un $52,5 \%$ afirmaron estar en total acuerdo con la afirmación desarrollada en este ítem, quedando un $5,5 \%$ en desacuerdo ya que para ellos los docentes no tienen esta característica. LIDERAZGO EFICIENTE: Se observa también, que el $50 \%$ de los estudiantes manifiestan estar en total acuerdo que sus docentes desarrollen eficazmente un liderazgo en su desempeño laboral y un $22,5 \%$ manifiestan estar en total desacuerdo ya que según ellos los docentes de la Facultad de Enfermería no son buenos líderes.

Tabla 1. Perfil de la muestra de estudiantes encuestados en la Facultad de Enfermería de la Universidad Nacional San Luis Gonzaga de Ica

\begin{tabular}{|c|c|c|c|c|c|c|c|c|}
\hline Perfil de la Muestra & II & III & IV & V & VI & VII & VIII & Tot. \\
\hline \multicolumn{9}{|l|}{ Edad } \\
\hline-20 & 18 & 14 & 15 & 12 & 14 & 6 & 5 & 84 \\
\hline $20-25$ & 8 & 9 & 3 & 5 & 9 & 19 & 20 & 73 \\
\hline $25-30$ & 3 & 5 & 3 & 8 & 8 & 3 & 2 & 32 \\
\hline+30 & 2 & - & 1 & 2 & 2 & 2 & 1 & 10 \\
\hline \multicolumn{9}{|l|}{ Sexo } \\
\hline Masculino & 1 & 3 & 2 & 3 & 3 & -- & -- & 12 \\
\hline Femenino & 30 & 26 & 20 & 24 & 30 & 30 & 28 & 188 \\
\hline \multicolumn{9}{|l|}{ Trabajo } \\
\hline $\mathrm{SI}$ & 3 & 3 & 5 & 5 & 3 & 2 & 3 & 24 \\
\hline $\mathrm{NO}$ & 28 & 26 & 17 & 22 & 30 & 28 & 25 & 176 \\
\hline \multirow{2}{*}{\multicolumn{9}{|c|}{$\begin{array}{l}\text { Situación de } \\
\text { Estudiantes }\end{array}$}} \\
\hline & & & & & & & & \\
\hline Regular & 28 & 23 & 19 & 24 & 30 & 28 & 25 & 177 \\
\hline Reingresante & 1 & 2 & 1 & 1 & - & - & 2 & 7 \\
\hline Repitente & 2 & 4 & 2 & 2 & 3 & 2 & 1 & 16 \\
\hline \multicolumn{9}{|l|}{ Estado Civil } \\
\hline Soltero & 28 & 20 & 21 & 251 & 29 & 28 & 25 & 176 \\
\hline Casado & 2 & 6 & - & 1 & 2 & 1 & 3 & 15 \\
\hline Conviviente & $\overline{1}$ & 3 & 1 & & 2 & 1 & - & 9 \\
\hline
\end{tabular}




\section{Tabla 2. Resultados sobre los estilos de liderazgo en los docentes de la Facultad de Enfermería de la UNICA}

\begin{tabular}{|c|c|c|c|c|c|c|c|c|c|c|}
\hline \multirow[t]{2}{*}{ TIPO DE ESTILO } & \multicolumn{2}{|c|}{$\begin{array}{l}\text { Totalmente de } \\
\text { acuerdo }\end{array}$} & \multicolumn{2}{|c|}{ De acuerdo } & \multicolumn{2}{|c|}{$\begin{array}{c}\text { Ni de acuerdo } \\
\text { ni en } \\
\text { desacuerdo }\end{array}$} & \multicolumn{2}{|c|}{$\begin{array}{c}\text { En } \\
\text { desacuerdo }\end{array}$} & \multicolumn{2}{|c|}{$\begin{array}{c}\text { Totalmente } \\
\text { en } \\
\text { desacuerdo }\end{array}$} \\
\hline & $\mathbf{N}^{\circ}$ & $\%$ & $\mathbf{N}^{\circ}$ & $\%$ & $\mathbf{N}^{\circ}$ & $\%$ & $\mathbf{N}^{\circ}$ & $\%$ & $\mathbf{N}^{\circ}$ & $\%$ \\
\hline $\begin{array}{l}\text { CARISMA } \\
\text { Considera que tus profesores son } \\
\text { amables y confiables }\end{array}$ & 66 & $33 \%$ & 108 & $54 \%$ & 12 & $6 \%$ & 2 & $1 \%$ & 12 & $6 \%$ \\
\hline $\begin{array}{l}\text { ESTIMULO INDIVIDUAL } \\
\text { Considera que tus profesores } \\
\text { propicien en los alumnos que } \\
\text { motivos para crear nuevas ideas }\end{array}$ & 26 & $13 \%$ & 130 & $65 \%$ & 22 & $11 \%$ & 4 & $2 \%$ & 18 & $9 \%$ \\
\hline $\begin{array}{l}\text { CONSIDERACIÓN INDIVIDUAL } \\
\text { Considera que tus profesores son } \\
\text { capaces de escucharlos cuando los } \\
\text { necesitas }\end{array}$ & 112 & $56 \%$ & 28 & $14 \%$ & 12 & $6 \%$ & 34 & $17 \%$ & 14 & $7 \%$ \\
\hline $\begin{array}{l}\text { TOLERANCIA PSICOLOGICA } \\
\text { Considera que tus profesores se } \\
\text { muestran tolerantes ante los errores } \\
\text { de sus alumnos }\end{array}$ & 56 & $28 \%$ & 92 & $46 \%$ & 8 & $4 \%$ & 22 & $11 \%$ & 22 & $11 \%$ \\
\hline $\begin{array}{l}\text { MOTIVACIÓN INSPIRADA } \\
\text { Considera que tus profesores suelen } \\
\text { ser optimistas y motivadores en su } \\
\text { desempeño laboral }\end{array}$ & 105 & $52,5 \%$ & 80 & $40 \%$ & 3 & $1,5 \%$ & 10 & $5 \%$ & 2 & $1 \%$ \\
\hline $\begin{array}{l}\text { CONSIDERACIÓN INDIVIDUAL } \\
\text { Considera que tus profesores tienen } \\
\text { en cuenta las necesidades } \\
\text { individuales de cada alumno }\end{array}$ & 84 & $42 \%$ & 72 & $36 \%$ & 10 & $5 \%$ & 22 & $11 \%$ & 12 & $6 \%$ \\
\hline $\begin{array}{l}\text { INFLUENCIA IDEALIZADA } \\
\text { Considera que tus profesores te } \\
\text { transmiten } \quad \text { entusiasmo y y } \\
\text { confianza }\end{array}$ & 105 & $52,5 \%$ & 35 & $17,5 \%$ & 45 & $22,5 \%$ & 11 & $5,5 \%$ & 4 & $2 \%$ \\
\hline $\begin{array}{l}\text { LIDERAZGO EFICIENTE } \\
\text { Considera que tus profesores } \\
\text { desarrollan un liderazgo eficaz en su } \\
\text { desempeño laboral }\end{array}$ & 100 & $50 \%$ & 35 & $17 \%$ & 10 & $5,5 \%$ & 10 & $5,5 \%$ & 45 & $22 \%$ \\
\hline
\end{tabular}

\section{DISCUSIÓN.}

Las dimensiones que se identificaron dentro de la escala de liderazgo docente es el estilo de liderazgo transformador.

Al analizar los resultados obtenidos del cuestionario aplicado a los estudiantes de la Facultad de Enfermería de la Universidad San Luis Gonzaga de Ica sobre la actuación desempeñada en su rol de líder, se pudo constatar que el porcentaje más alto está en respuestas positivas, afirmando que los docentes desarrollan en su desempeño características de liderazgo apegados a los lineamientos propuesto en la teoría de Bass, relacionado con el liderazgo transformacional (5).

Se encuentra que los estudiantes consideran que sus docentes se muestran amables y confiables, cualidad que permite mantener comunicaciones efectivas con sus alumnos y con las personas que los rodean. Cabe resaltar que si es cierto sus respuestas son afirmativas no están dentro del "Totalmente de Acuerdo" como si todavía no están convencidos totalmente del actuar de su docente todavía muestran ambigüedades en sus respuestas y esto es preocupante porque es importante destacar que el carisma es una de las competencias que todo docente líder debería manejar a plenitud, ya que es la base fundamental de las relaciones interpersonales.

La motivación inspiradora el líder crea una visión estimulada y atractiva para sus seguidores, los docente de la Facultad de Enfermería el $65 \%$ de encuestados está de acuerdo en afirmar que sus docentes demuestran su compromiso personal y entusiasmo por la visión para conseguir entusiasmos y arrastrar a los demás. 
En este sentido debemos observar esta característica como diagnóstico de las necesidades individuales de los docentes y alentarlos individualmente.

El estímulo intelectual promueve al docente a buscar nuevos enfoques y nuevas soluciones a los problemas. En tal sentido los alumnos manifiestan que el docente de la Facultad de Enfermería promueve en sus alumnos la estimulación intelectual este aspecto esencial reside en proponer nuevos enfoques para viejos problemas; es decir el educador debe hacer énfasis en la inteligencia y racionalidad para la solución de los problemas (9).

El docente líder debe tener en cuenta las necesidades individuales de cada alumno para guiar a cada uno a seguir sus potencialidades. El docente debe actuar como entrenador (coach) abriendo oportunidades de aprendizaje y creando un clima de apoyo; es un líder que escucha y sabe delegar, dando luego FeedBack constructivo al subordinado.

Coherente con el modelo planteado es muy importante considerar la tolerancia psicológica porque esto implica hacer uso del buen sentido del humor para indicar equivocaciones, para resolver conflictos $y$ para manejar momentos duros. Esto es la que Bass denomina la tolerancia psicológica como proceso de aprender a tolerar los errores de los demás (5).

\section{CONCLUSIONES}

Después del análisis de todos los datos se concluye que:

- Existe un buen desempeño laboral del docente de la Facultad de Enfermería, también se evidencia que existe nudos críticos que deben ser atendidos para dar respuesta satisfactoria a los estudiantes,

- Que a su vez los docentes actúan de manera que los estudiantes están de acuerdo con el liderazgo que ejerce a sus docentes y que quieren imitar su entusiasmo con la idea de que en el futuro serán como sus docentes (Bernal 2000).

- Los estudiantes perciben que el liderazgo de los docentes de la Facultad de Enfermería es el Transformacional.

Se evidencia que los docentes de la Facultad de Enfermería desarrollan un estilo de liderazgos adecuados relacionados con la teoría presentada por Bass donde las mayores respuestas reflejan un estilo basado en liderazgo transformacional donde muestran características como: carisma, consideración individual, respeto al prójimo buscando fortalecer los valores que permiten una actuación creativa, independiente y autorealizante.

\section{Correspondencia:}

Rosa Hernández Onofra

Correo Electrónico: rosaherono@hotmail.com

\section{REFERENCIAS BIBLIOGRAFICAS}

1. García, E. Liderazgo Docente. Universidad Nacional Autónoma de México "UNAM". Revista Latindex UNAM. 2006. [Internet] Disponible en: http://www.prog-edu.org/ forodelideres/empezar aqui archivos/lider azgo-docente.pdf

2. Flores, R. Percepciones que tienen los profesionales de la educación. Revista de Orientación Educacional IRESIE, México. 1998. [Internet] Disponible en: http://educacion.upa.cl/decano/ percepcion.htm (enero de 2007)

3. Bernal, J. Liderar el cambio: El liderazgo transformacional. Anuario de Pedagogía. Volumen 2 (197 - 230). Departamento de Ciencias de la Educación. Universidad de Zaragoza, España. 2000.

4. Grinberg J. Desafíos y posibilidades para el futuro de la educación. Universidad Torcuato Di Tella. Primer Coloquio, México. 1999

5. Bass, B. Leadership and performance beyond espectations. New York, United States. The Free Press. 1985 
6. Padilla D. "Percepción de los Docentes, Administrativos y Alumnos sobre el Liderazgo y Cultura Organizacional en la Universidad Privada Antenor Orrego, Trujillo, Perú. 2005. [Internet] Disponible en: http://www.yasni. es/ext.php?url=http $\% 3 \mathrm{~A} \% 2 \mathrm{~F} \% 2 \mathrm{Fwww}$.buenastareas.com\%2F ensayos\%2FAlalsitras\%2F2330105.html\& name $=$ Dante + Padilla\&cat $=$ document $\&$ sho wads $=1$

7. Rodríguez N. "El Clima Escolar". Revista Digital "Investigación y Educación". Rv. N 7, Vol. 3. España, 2005. [Internet] Disponible en:

http://www.csif.es/archivos migracion estr uctura/andalucia/modules/mod sevilla/arch ivos/revistaense/n7v3/clima.PDF
8. Arias, F. El proyecto de Investigación. Caracas, Venezuela: Episteme. Primera Edición. 2004.

9. Gutiérrez, M. Liderazgo transformacional en el docente universitario. Universidad de Panamá. Volumen №1. 2003.

Recibido: 10/02/14

Aprobado para Publicación: 18/05/14 